\title{
Thermal analysis of the LLR optical telescope tube assembly based in Hartebeesthoek Radio Astronomy Observatory
}

\author{
P. Tsela ${ }^{1}$ (D) L. Combrinck ${ }^{1,2} \cdot$ R. Botha ${ }^{2} \cdot$ B. Ngcobo $^{3}$
}

Received: 20 November 2014/ Accepted: 30 June 2015/Published online: 17 July 2015

(C) Akadémiai Kiadó 2015

\begin{abstract}
The Hartebeesthoek Radio Astronomy Observatory of South Africa is currently developing a lunar laser ranger (LLR) system based on a one metre aperture telescope in collaboration with National Aeronautics and Space Administration and the Observatoire de la Côte d'Azur. This LLR will be an addition to a limited list of operating LLR stations globally and it is expected to achieve sub-centimetre range precision to the Moon. Key to this expectation including the overall telescope operational performance is thermal analysis of the telescope structure, based on the thermal properties of component materials and their interaction with the environment through conventional heat transfer mechanisms. This paper presents transient thermal simulation results of the telescope's optical tube and one metre primary mirror in terms of thermal variations and consequent structural deformations. The results indicate that on a non-windy, cloud-free and winter day, the temperature gradients on the structure could be within $1{ }^{\circ} \mathrm{C}$ with respect to the temporal ambient air temperatures at the site when these are between 9 and $23{ }^{\circ} \mathrm{C}$. Furthermore, these gradients were coupled with thermally-induced total deformations that vary between 2.9 and $40.7 \mu \mathrm{m}$ of the assembled telescope components. In overall, these findings suggest that both the tube and especially the mirror may respond very slowly to ambient temperatures; however, correcting for structural thermal variations is imperative in maximizing the pointing accuracy of the telescope thereby increasing the chance being on-target with the retroreflectors located on the Moon surface.
\end{abstract}

Keywords Thermal variation - Deformation - Optical tube · Primary mirror · Telescope pointing

P. Tsela

philemon.tsela@up.ac.za

1 Department of Geography, Geoinformatics and Meteorology, University of Pretoria, Pretoria, South Africa

2 Space Geodesy Programme, Hartebeesthoek Radio Astronomy Observatory, Krugersdorp, South Africa

3 Department of Physics, University of Pretoria, Pretoria, South Africa 


\section{Introduction}

Over the past decades, studies have demonstrated that an optical telescope operating at an observatory site is subject to a varying thermal environment which influences the thermal behaviour of the telescope structure and its optical performance (Perry 1943). At an observatory site, an optical telescope interacts with solar radiation, ground surface, sky and ambient air temperatures (Greve and Bremer 2010), thus the relative effects induced by each of these parameters on the operational and optical performance of the telescope varies both spatially and temporally. In particular, the changing ambient air temperature (hereafter $T_{\infty}$ ) cause a transfer of energy by e.g., convection (i.e., between the telescope and its surrounds) and conduction (i.e., between the telescope component materials) thereby changing the thermal state of the telescope. The thermal variations on the telescope due to $T_{\infty}$ should be well understood in order to determine (i) their effect on the pointing of the telescope and (ii) options for developing a thermal dynamic model which would correct for thermal variations.

A thorough understanding of thermal variations on a telescope is incurred using two widely known approaches. The first approach requires the installation of a network of thermal sensors particularly on the identified major component materials of a telescope in order to obtain real measurements of thermal variations (Bely 2003; Greve and Bremer 2010). The alternate approach entails simulating thermal variations based on a geometrically accurate three-dimensional model of a telescope, including its corresponding thermal properties of the component materials (Bely 2003; Greve and Bremer 2010). However, it has been reported that the reliability of thermal simulation outputs depends to a large extent on the correct parameterisation of thermal loads which include e.g., $T_{\infty}$, convection coefficient $(h)$ of air temperature and material thermal properties (Cho et al. 2009; Cho et al. 2010), and thus if possible may have to be validated using real measurements. In this study the latter approach was adopted for the analysis of thermal variations as well as related deformations on the lunar laser ranger (LLR) optical telescope structure based at Hartebeesthoek Radio Astronomy Observatory (HartRAO) located in South Africa.

Numerous studies have used the latter approach to determine the effects of thermal variations and related deformations on the pointing of an optical telescope. For example, Cho et al. (2010) conducted a thermal analysis of the thirty meter telescope (TMT) structure under different predetermined thermal loads for optical performance over day and night temperatures spanning three consecutive days. The study discovered structural thermal variations ranging between 0.01 and $7.32{ }^{\circ} \mathrm{C}$ corresponding with thermally-induced deformations ranging between 141 and $993 \mu \mathrm{m}$ respectively (Cho et al. 2010), and thus were shown to have a temporal impact on telescope pointing with offsets ranging from $0.7^{\prime \prime}$ to $1^{\prime \prime}$ at selected elevation angles (Vogiatzis et al. 2014). Mittag et al. (2008) analysed the influence of ambient air temperatures on the pointing of the Hamburg robotic optical telescope for 16 nights (with temperatures ranging from -6.4 to $25.8{ }^{\circ} \mathrm{C}$ ) and found that thermal expansion of component materials primarily triggered misalignments of the optical axis with the tube. This expansion was responsible for the observed peak azimuth and elevation offsets of $40^{\prime \prime}$ and $10^{\prime \prime}$ respectively. Furthermore, a number of studies have also demonstrated virtually similar effects of thermal variations and resulting deformations on the pointing of radio telescopes e.g., Combrinck and Merry (1997), Bremer and Penalver (2002), Shinnage et al. (2004), Greve et al. (2005) and Wresnik et al. (2007). In overall, these studies agree that the thermal behaviour of a site-based telescope could have an impact on the required telescope performance (e.g., pointing) and should therefore be well 
understood with respect to the ambient climatic environment, design and thermal properties of component materials.

Therefore, this study presents a simulation-based thermal analysis of the HartRAO LLR telescope component materials in terms of thermal variations and induced deformations. The simulation was performed in ANSYS (Lawrence 2012) and provided a simulation of the thermal behaviour of the HartRAO LLR telescope, particularly with respect to its thermal material properties and $T_{\infty}$. The ultimate objective, which is beyond the scope of this paper, is to develop a dynamic thermal model that can mitigate the thermal variations on the entire telescope to $\leq 1{ }^{\circ} \mathrm{C}$ and subsequently, feed these corrections into a telescope pointing model (currently under development in HartRAO) to counteract resulting pointing offsets. With regards to optimal thermal modelling of a telescope, it is worth mentioning that a major challenge is to develop solution strategies that can mitigate and stringently regulate the thermal variations to $\sim 1{ }^{\circ} \mathrm{C}$ (Murphy et al. 2008).

\section{System requirements}

The overall goal of this study is to have a dynamic thermal model that can maintain the thermal variations of critical telescope component materials i.e., optics and tube support structure to $\sim 1{ }^{\circ} \mathrm{C}$ by using measurements obtained from an expandable number $n$ of thermal sensors $T_{n}$. These thermal variations should be measured and regulated in real time in order to stringently alleviate the induced expansion and misalignment of component materials. The thermal model is expected to contribute toward the attainment of the proposed pointing and optical requirements of the LLR telescope (HartRAO 2005; Combrinck 2011; Combrinck and Botha 2014) during normal operations, which are summarized in Table 1.

The requirements of the HartRAO LLR telescope (Table 1) are crucial in the correct and comprehensive interpretation of the thermal modelling. In particular, this study, sought to understand thermal variations and deformations of the critical telescope components due to $\mathrm{T}_{\infty}$ and their likelihood to compromise the pointing accuracy (Table 1) of the telescope during ranging. Furthermore, it is imperative to exhaust the investigation regarding the influence of thermal effects on other parameters of the telescope requirements (Perry 1943) as this would guide the development of robust correction techniques particularly for structural thermal variations.

Table 1 Summary of the selected pointing and optical constraints and requirements of the HartRAO LLR telescope

\begin{tabular}{lc}
\hline Pointing related & Optical related \\
\hline Range precision: $1 \mathrm{~mm}$ & Transmit/receive efficiency: $>0.9 />0.8$ \\
Pointing accuracy: $\leq 0.5^{\prime \prime}$ & Estimated photons: $3-4$ per minute based on the proposed \\
& $130 \mathrm{~mJ}, 80$ pico-second pulse width system and an \\
& atmospheric seeing condition of $2-3^{\prime \prime}$ \\
Tracking accuracy: $<1^{\prime \prime}$ & Return detector: avalanche photo diodes with $100-200$ micron \\
& aperture and detection efficiency of $\sim 50 \%$. Single photon \\
& mode with a wavelength of $1064 \mathrm{~nm}$ \\
Maximum slew rate in azimuth and & Start detector fast ( $<50$ Pico seconds) photo diode detecting at \\
elevation: $\sim 3$ degrees per second & 532 nm wavelength. Single or multiphoton mode \\
Position resolution: $<0.5^{\prime \prime}$ & Effective focal length: $7.6 \mathrm{~m}$ \\
\hline
\end{tabular}

The two columns do not relate to each other 


\section{Materials used}

\subsection{Thermal properties of component materials and air temperature variations}

Various components of the LLR telescope based at HartRAO are currently being integrated and coupled with the development of models for operating the instrument (Combrinck and Botha 2014). Figure 1 presents a 3-D drawing prepared in SolidWorks (Planchard and Planchard 2013) which depicts selected structural components of the telescope. Structural components such as these are deemed sensitive to $T_{\infty}$ and thus significant for pointing accuracy (Perry 1943). In addition, the thermal properties related to each component material are also presented (Table 2 ) and play a significant role in the correct parameterisation of the thermal model.

Take note, the structural components are shown in exploded view according to Fig. 1 simply for legibility, however these components were considered as one assembled structure during thermal analysis (i.e. a fully assembled optical tube). From Table 2 it can be seen that the primary mirror material i.e. Zerodur, has the lowest thermal conductivity $(k)$ and coefficient of thermal expansion (CTE) compared to all other material components of the telescope (Schott 2011).

Therefore, it is clear that the primary mirror might result in slow internal equalization of thermal variations coupled with very small distortions (Jedamzik et al. 2010) relative to other material components; particularly with respect to HartRAO site-based air temperature profiles which are on average broadly estimated in the range $0{ }^{\circ} \mathrm{C}$ to $\pm 35{ }^{\circ} \mathrm{C}$ (Fig. 2).

\section{Methods used}

\subsection{Analysis of thermal variations}

A temporal analysis of thermal variations on the telescope components (Fig. 1) was performed in ANSYS software based on traditional heat transfer mechanisms expressed in Eqs. (1) and (2).

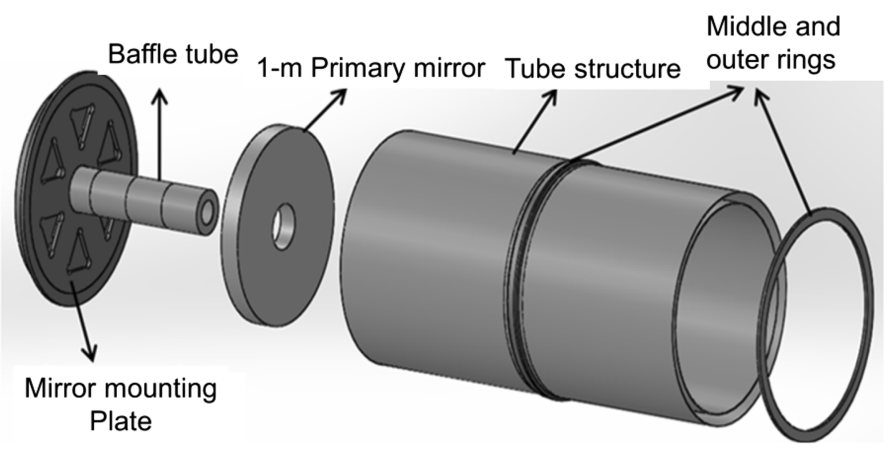

Fig. 1 A 3-D model of the HartRAO LLR telescope optical tube assembly, illustrating the major structural components 
Table 2 The thermal properties of the telescope component materials (Green 2008)

\begin{tabular}{llll}
\hline & $\begin{array}{l}\text { Primary mirror } \\
\text { (Zerodur) }\end{array}$ & $\begin{array}{l}\text { Tube structure; baffle } \\
\text { tube (Aluminium) }\end{array}$ & $\begin{array}{l}\text { Mirror mounting plate; middle } \\
\text { and outer rings (Mild steel) }\end{array}$ \\
\hline $\begin{array}{l}\text { Thermal Conductivity } \\
k(\mathrm{~W} / \mathrm{m} \mathrm{K})\end{array}$ & 1.46 & 130 & 50 \\
$\begin{array}{l}\text { Specific heat } C_{p}(\mathrm{~J} / \mathrm{kg} \mathrm{K}) \\
\text { CTE } 10^{-6} \mathrm{~K}^{-1}\end{array}$ & 820 & 1047 & 490 \\
Density $p\left(\mathrm{~kg} / \mathrm{m}^{3}\right)$ & 0.10 & 24.4 & 11.7 \\
\hline
\end{tabular}

$$
\begin{gathered}
\vec{Q}_{\text {conv }}=h A_{x}\left(T_{x}-T_{\infty}\right) \vec{i}+h A_{y}\left(T_{y}-T_{\infty}\right) \vec{j}+h A_{z}\left(T_{z}-T_{\infty}\right) \vec{k} \\
\vec{Q}_{\text {cond }}=-k\left(A_{x} \frac{\partial T}{\partial x} \vec{i}+A_{y} \frac{\partial T}{\partial y} \vec{j}+A_{z} \frac{\partial T}{\partial z} \vec{k}\right)
\end{gathered}
$$

In Eq. (1) $\vec{Q}_{\text {conv }}$ represents the convection heat transfer rate between the telescope components and the varying daily ambient temperature $\left(T_{\infty}\right)$ at HartRAO. Here, $h$ denote the convection coefficient of air temperature; $\vec{i}, \vec{j}$ and $\vec{k}$ denote the unit vectors; $A_{x}, A_{y}$ and $A_{z}$ represent the surface areas; $T_{x}, T_{y}$ and $T_{z}$ are the temperatures of the corresponding surfaces in three dimensional space of the assembled telescope structure respectively (Cengel and Ghajar

2011). Furthermore, in Eq. (2) $\vec{Q}_{\text {cond }}$ represent the conduction heat transfer rate through the telescope component materials as a result of, their temperature difference $\partial T$ in three dimensional space; and the thermal conductivity $k$ (Cengel and Ghajar 2011).

In particular, the assembled telescope structure (Fig. 1) was subjected to an initial temperature of $9{ }^{\circ} \mathrm{C}$ and subsequently exposed to $T_{\infty}$ typical of the HartRAO site during the time period 00:00 and 11:30 am for a particular day in June. In this study, we assumed stationary air at room temperature around the telescope structure by adopting a constant $h$ value of $0.025 \mathrm{~W} / \mathrm{m}^{2} \mathrm{C}$. This assumption thus excludes the wind effect during this thermal analysis. The wind effect including the varying $h$ values representative of natural and/or forced convection are possible future considerations for the current analysis so as to approximate reality as far as possible. In overall, this analysis provided insight about the thermal variations on and between the telescope components, particularly how these components thermally responded to $T_{\infty}$.

\subsection{Analysis of thermally-induced deformations}

The analysis of thermal deformations of the telescope component materials were calculated primarily based on material length and volume changes due to thermal variations, expressed as $\alpha_{l} \Delta T$ and $\alpha_{v} \Delta T$ in Eqs. (3) and (4) respectively (Dalcher et al. 1977).

Change in length $(\mu \mathrm{m})$ with temperature:

$$
\frac{l_{f}-l_{o}}{l_{o}}=\alpha_{l}\left(T_{f}-T_{o}\right)=\alpha_{l} \Delta T .
$$

Change in volume $\left(\mathrm{m}^{3}\right)$ with temperature:

$$
\frac{v_{f}-v_{o}}{v_{o}}=\alpha_{v}\left(T_{f}-T_{o}\right)=\alpha_{v} \Delta T
$$



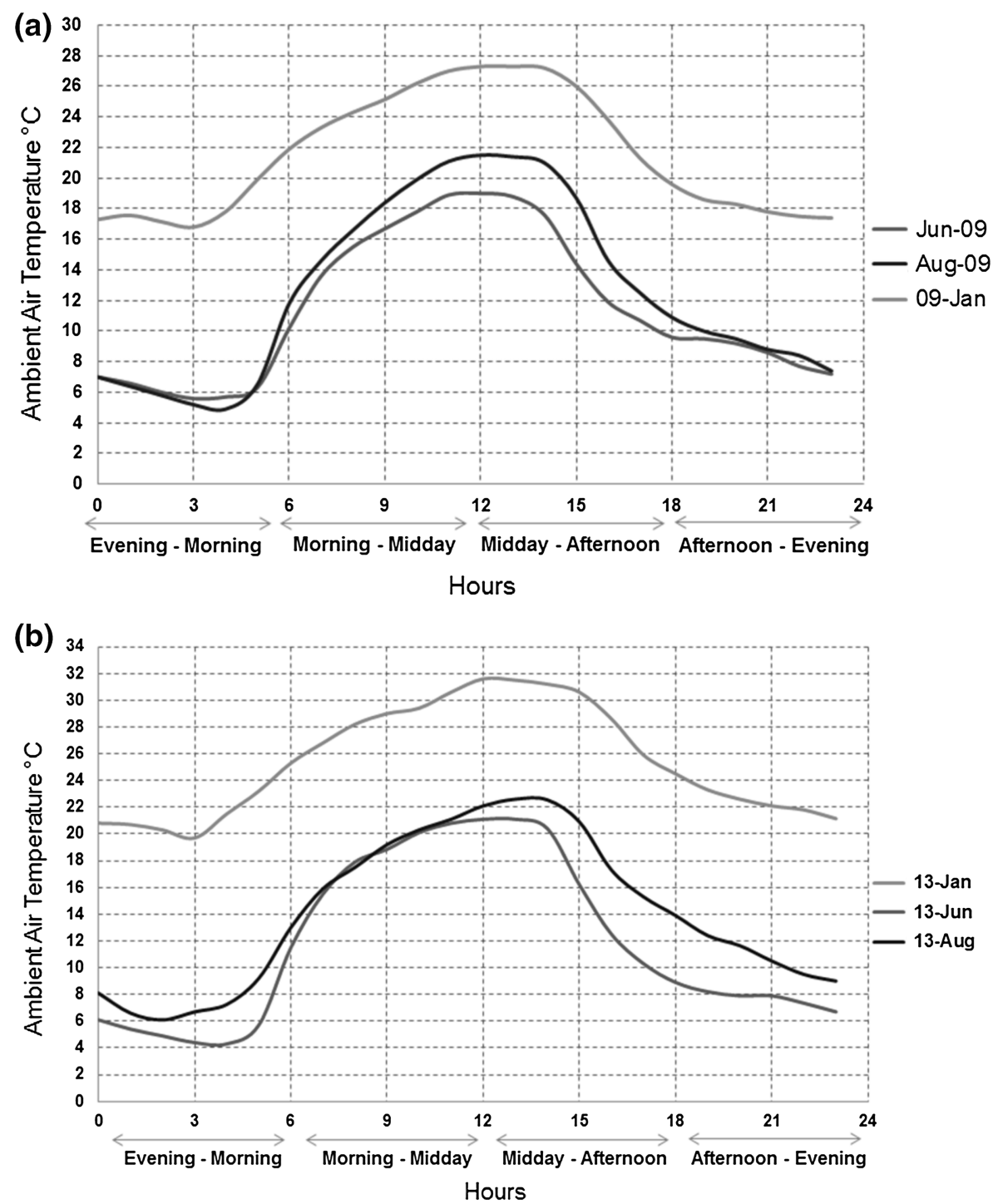

Fig. 2 Hourly diurnal-air-temperature changes at the HartRAO site for summer, winter and autumn seasons of 2009 (a) and 2013 (b). These temperature plots were used to guide the estimation of the widest possible temperature range including the approximate times when rapid temperature changes occur at the site where the LLR telescope is located

In Eq. 3, the symbol $\alpha_{l}$ represents the linear CTE which quantifies the amount of strain on a material with a change in temperature $\Delta T$. The symbol $\alpha_{v}$ in Eq. (4) denotes the volume CTE or the extent to which the volume of a material changes subject to $\Delta T$. This analysis was conducted using the ANSYS software package to provide a spatial and temporal indication of the total thermal deformations of the telescope component materials in the $\mathrm{x}, \mathrm{y}$ and $\mathrm{z}$ directions. 


\section{Results and discussion}

\subsection{Analysis of thermal variations}

For $T_{\infty}$ that varied from $\sim 9$ to $23{ }^{\circ} \mathrm{C}$ spanning the time period 00:00 and 11:30 a.m., the resulting minimum and maximum thermal variations across the telescope component materials were 9.3 to $10.1{ }^{\circ} \mathrm{C}$ (Fig. 3). In particular, the (Zerodur) primary mirror had the lowest thermal variations of $\sim 0.1{ }^{\circ} \mathrm{C}$ relative to the other component materials with respect to $T_{\infty}$ for the time period under study. This observation is consistent with the thermal properties of the mirror (see, Table 2 and Schott 2011), especially for low $k$; thus its slow thermal response to $T_{\infty}$ fulfils our requirement of regulating the thermal variations to $\leq 1{ }^{\circ} \mathrm{C}$. However, timely equalization (by using forced air ventilation) of these variations on the mirror (which responds comparatively slow to ambient temperature changes) including other component materials is important for overall optical and pointing performance of the telescope (Perry 1943).

Further, a slight increase of $\sim 0.23{ }^{\circ} \mathrm{C}$ of the thermal variations is seen on the steel mounting plate where the mirror rests. The minimal variations revealed by the two aforementioned components are in contrast with those on the tube structure (Fig. 3). The tube structure including the baffle tube (which are both of aluminium) had the highest thermal variations in the range $9.54-10.12{ }^{\circ} \mathrm{C}$ with respect to $T_{\infty}$. In particular, the baffle tube is surrounded by a very good insulator (i.e. Zerodur mirror material) which reduces heat transfer at the bottom side of the tube; this as a result, makes the top part of the baffle tube to be more exposed to ambient air hence it appears warmer. Additionally, the isotherms along the tube suggest that the thermal variations could be unidirectional, propagating from the 'open' front-end of the tube toward the 'closed' back-end where the primary mirror and mounting plate are structurally located. The evident decreasing temperatures toward the 'closed' back-end of the tube, suggest continuous (natural) heat losses as the thermal energy propagates along the optical tube in a unidirectional manner. These heat losses along the tube could have been accelerated by the contacting middle outer ring surface which is made of steel, coupled with dissimilar thermal properties relative to the aluminium tube.

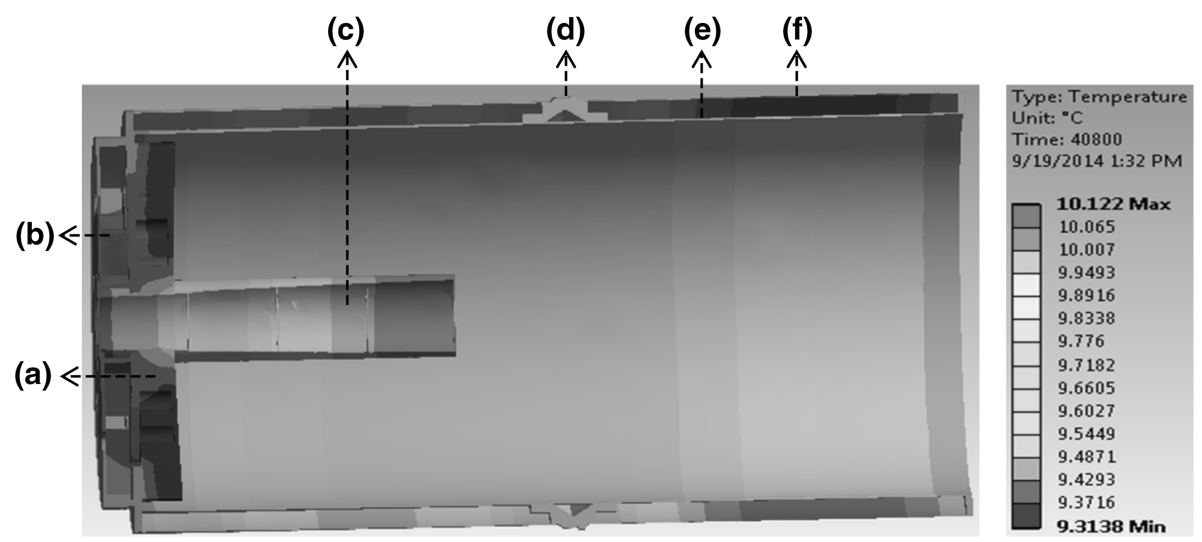

Fig. 3 Cross-section of the assembled telescope component materials showing the simulated thermal variations as modelled by ANSYS. The symbols $a, b, c, d, e$ and $f$ denotes the primary mirror, mounting plate, baffle tube, middle outer ring, inner tube surface and outer tube surface respectively 
Take note that the tube design consists of two layers or surfaces, i.e., the outer shell and inner shell (both made of thin aluminium; thus leaving an open region between the surfaces for insulation purposes. This is evident in Fig. 3 where the relative thermal gradients on the inner and outer shells respectively can be seen clearly. Furthermore, the thermal variations probed specifically from the mirror and optical tube surfaces revealed a total temperature gradient of $\sim 1{ }^{\circ} \mathrm{C}$ (Fig. 4), meaning that we can generally expect these component materials to respond very slowly to $T_{\infty}$. However, thermal regulatory measures may be necessary (this will be done through a microprocessor controlled venting system) to ensure these variations are equalized and kept to a minimum as much as possible i.e., $<1{ }^{\circ} \mathrm{C}$ (Murphy et al. 2008) to allow excellent telescope pointing, thereby increasing the chance of being on-target with the retroreflectors located on the lunar surface.

\subsection{Analysis of thermally-induced deformations}

The thermal analysis results presented in Fig. 3 were used to estimate the total thermallyinduced deformations of the telescope structure in $\mathrm{x}, \mathrm{y}$ and $\mathrm{z}$ directions (Fig. 5). Figure 5 illustrates the total deformations in the range $2.9-40.7 \mu \mathrm{m}$ spanning the time period 00:00 and 11:30 a.m.. In particular, the resistance against $\Delta T$ by the mirror shows virtually zero localised deformations of the mirror as well as its mounting plate (Fig. 5). These observations concur with modelling studies on the thermal expansion of Zerodur glass material at varying temperature profiles (i.e., between 0 and $50{ }^{\circ} \mathrm{C}$ ) which showed no radial variations and excellent homogeneity (Jedamzik et al. 2010). However, the magnitude of the error contribution from derived deformations of the mirror is yet to be investigated for telescope pointing at HartRAO.

The effects of the derived thermal variations (Fig. 3) are largely evident along the edges of the tube surface through material expansion (Fig. 5); and thus, have been reported elsewhere to be a significant source for a misalignment of the optical axis to the telescope tube axis (see Mittag et al. 2008 and Shinnaga et al. 2004). In this context, the optical axis entails the correct position of the spider assembly carrying the secondary focus mirror (not shown in Fig. 5) in relation to that of the primary mirror. The secondary mirror is attached to the front of the optical tube using a spider assembly (this allows centring and focussing). Therefore, any slight deformations on the mirrors and/or materials holding the mirrors would potentially misalign the optical axis thereby contributing to pointing errors and

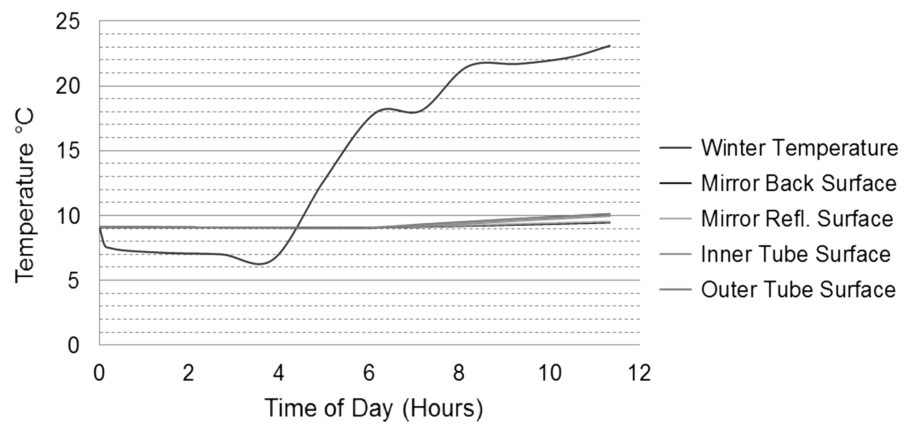

Fig. 4 Temporal thermal variations on the tube and mirror surfaces in relation to $T_{\infty}$ typical of the HartRAO site, during the time period 00:00 and 11:30 am for a particular day in June. The thermal variations were probed from Fig. 3 only for the abovementioned surfaces 


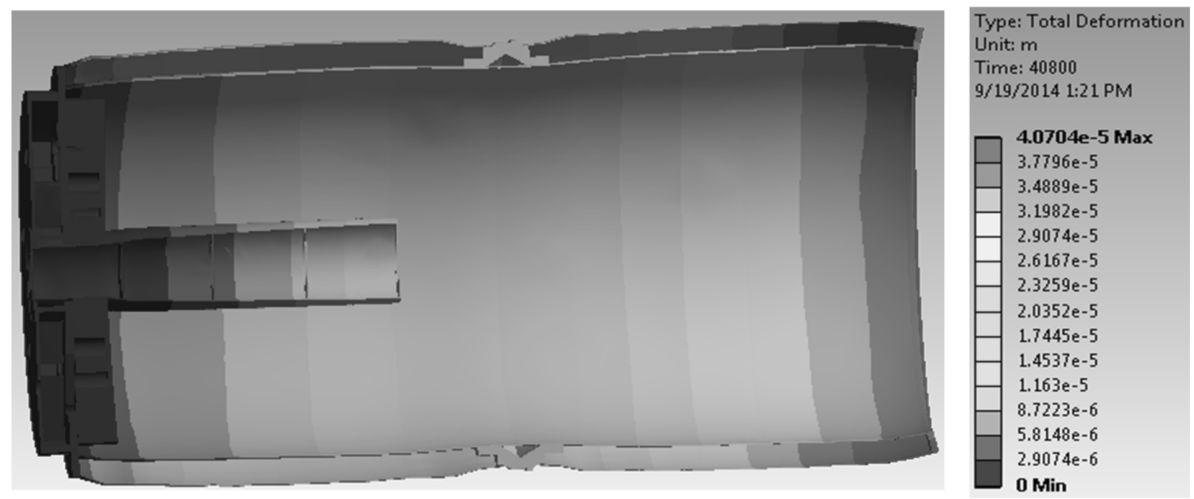

Fig. 5 A cross-section of the telescope component materials showing the thermally-induced structural deformations in $\mathrm{x}, \mathrm{y}$ and $\mathrm{z}$ directions

focussing problems during ranging. These findings call for the development of a thermal dynamic model which can compensate for structural misalignments due to thermal variations.

\section{Conclusions}

This study has performed a first-step thermal analysis on selected component materials comprising the HartRAO LLR optical telescope tube assembly, with the aim of simulating and understanding its thermal behaviour. The analysis entailed parameterisation of heat transfer equations in ANSYS software to derive thermal variations and related deformations of the telescope component materials, in relation to the varying ambient air temperatures at the HartRAO site. Overall, the results have shown total thermal variations of about $1{ }^{\circ} \mathrm{C}$; however, this could be due to the assumption (during parameterisation) that all component materials have a constant $h$ value of $0.025 \mathrm{~W} / \mathrm{m}^{2} \mathrm{C}$ at room temperature. Furthermore, this study will be expanded to investigate the effect of natural and forced convection with varying $h$ values on the telescope thermal variations. In addition, this analysis will be expanded to the other remaining telescope component materials particularly, the spider assembly, the secondary mirror, telescope azimuth-elevation mount, as well as base structures. These preliminary findings provide an indication of: (i) understanding the thermal behaviour of the telescope tubes critical components with respect to the changing thermal environment, (ii) guiding the strategic location of the temperature sensors on the telescope to obtain real thermal measurements (e.g., this could enable realtime derivation and prediction of structural deformations), and (iii) options for developing a thermal dynamic model which would correct for thermal variations that affect the pointing of the telescope. It is worth mentioning that relatively low-cost solutions are available on the market such as, thermal imagers, microelectromechanical systems (MEMS) thermal sensors and accelerometers for measuring temperature variations and structural deflections in real-time (Hirt and Seeber 2008; Pisanu et al. 2010; Mészáros et al. 2014). Such options may provide the opportunity to explore alternative solution strategies for implementing robust real-time thermal monitoring systems. 
Acknowledgments P. L. Tsela hereby acknowledges research support in part by the National Research Foundation of South Africa for the grant, Unique Grant No. 93952. Any opinion, finding and conclusion or recommendation expressed in this material is that of the author(s) and the NRF does not accept any liability in this regard. Further, we express our gratitude to the Inkaba yeAfrica project, the Department of Science and Technology of South Africa and HartRAO for support. We also acknowledge support from the Mechanical Engineering Department at the University of Pretoria for providing access to ANSYS software.

\section{References}

Bely P (2003) The design and construction of large optical telescopes. Springer, New York

Bremer M, Penalver J (2002) FE model-based interpretation of telescope temperature variations. In: Workshop on integrated modelling of telescopes. International Society for Optics and Photonics, pp 186-195

Cengel YA, Ghajar AJ (2011) Heat and mass transfer: fundamentals \& applications. McGraw-Hill, New York

Cho M, Corredor A, Vogiatzis K, Angeli G (2009) Thermal performance prediction of the TMT telescope structure. In: SPIE Optical Engineering+ Applications. International Society for Optics and Photonics, pp 74270E-74215E

Cho M, Corredor A, Vogiatzis K, Angeli G (2010) Thermal analysis of the TMT telescope structure. In: SPIE Astronomical Telescopes+ Instrumentation. International Society for Optics and Photonics, pp 77380C-77312C

Combrinck L (2011) Development of a satellite and lunar laser ranger and its future applications in South Africa. In: Proceedings of the 62nd international astronautical congress. Oct 2011, Cape Town, available at: http://iafastro.directory/iac/archive/browse/IAC-11/A2/1/

Combrinck L, Botha R (2014) Challenges and progress with the development of a Lunar Laser Ranger for South Africa. In: Proceedings of the 18th international workshop on laser ranging: pursuing ultimate accuracy and creating new synergies, 11-15 Nov 2013, Fujiyoshida, available at: http://cddis.gsfc.nasa. gov/lw18/docs/papers/Session13/13-05-04-Combrinck.pdf

Combrinck WL, Merry CL (1997) VLBI Antenna axis offset and intersection determination using GPS. J Geophys Res 102(B11): 24741-24743

Dalcher A, Yang T, Chu C (1977) High temperature thermal-elastic analysis of dissimilar metal transition joints. J Eng Mater Technol 99(1):65-69

Green DW (2008) Perry's chemical engineers' handbook. McGraw-hill, New York

Greve A, Bremer M (2010) Thermal design and thermal behaviour of radio telescopes and their enclosures. Springer, Berlin

Greve A, Bremer M, Penalver J, Raffin P, Morris D (2005) Improvement of the IRAM 30-m telescope from temperature measurements and finite-element calculations. IEEE Trans Antennas Propag 53(2): $851-860$

Hartebeesthoek Radio Astronomy Observatory (HartRAO) (2005) Proposed re-location and conversion of CNES SLR system to South Africa in collaboration with OCA and the greater ILRS community. Available at http://www.hartrao.ac.za/iisgeo/docs/LLR_proposal.doc

Hirt C, Seeber G (2008) Accuracy analysis of vertical deflection data observed with the Hannover Digital Zenith Camera System TZK2-D. J Geodesy 82(6):347-356

Jedamzik R, Johansson T, Westerhoff T (2010) Modeling of the thermal expansion behaviour of ZERODUR at arbitrary temperature profiles. In: SPIE Astronomical Telescopes+ Instrumentation. International Society for Optics and Photonics, pp 77390I-77312

Lawrence KL (2012) ANSYS workbench tutorial release 14. SDC Publications, Mission

Mészáros L, Jaskó A, Pál A, Csépány G (2014) Accurate Telescope Mount Positioning with MEMS Accelerometers. arXiv preprint arXiv:1407.0035

Mittag M, Hempelmann A, Gonzalez-Perez J, Schmitt J (2008) The temperature dependence of the pointing model of the hamburg robotic telescope. Publ Astron Soc Pac 120(866):425-429

Murphy Jr T, Adelberger EG, Battat J, Carey L, Hoyle CD, LeBlanc P, Michelsen E, Nordtvedt K, Orin A, Strasburg JD (2008) The apache point observatory lunar laser-ranging operation: instrument description and first detections. Publ Astron Soc Pac 120(863):20-37

Perry J (1943) Thermal effects upon the performance of lens systems. Proc Phys Soc 55(4):257

Pisanu T, Buffa F, Morsiani M, Pernechele C, Poppi S (2010) Thermal behavior of the Medicina 32-meter radio telescope. In: SPIE Astronomical Telescopes+ Instrumentation. International Society for Optics and Photonics, pp 773935-773939 
Planchard DC, Planchard MP (2013) SolidWorks 2013 Tutorial. SDC Publications, Mission

Schott A (2011) ZERODUR ${ }^{\circledR}$-Zero Expansion Glass Ceramic. Data sheet, Mainz

Shinnaga H, Chamberlin R, Phillips T, Dowel D, Yoshida H, Peng R (2004) A consideration of thermal effect on pointing measured on a Nasmyth focus of the CSO $10.4 \mathrm{~m}$ Leighton telescope at $\lambda 350 \mu \mathrm{m}$. Technical memorandum

Vogiatzis K, Sadjadpour A, Roberts S (2014) TMT telescope structure thermal model. In: SPIE Astronomical Telescopes+ Instrumentation. International Society for Optics and Photonics, pp 915011-915012

Wresnik J, Haas R, Boehm J, Schuh H (2007) Modeling thermal deformation of VLBI antennas with a new temperature model. J Geodesy 81(6-8):423-431 